\title{
Beam shaping to improve the free-electron laser performance at the Linac Coherent Light Source
}

\author{
Y. Ding, ${ }^{*}$ K. L. F. Bane, W. Colocho, F.-J. Decker, P. Emma, J. Frisch, M. W. Guetg, \\ Z. Huang, R. Iverson, J. Krzywinski, H. Loos, A. Lutman, T. J. Maxwell, H.-D. Nuhn, \\ D. Ratner, J. Turner, J. Welch, and F. Zhou \\ SLAC National Accelerator Laboratory, Menlo Park, California 94025, USA
}

(Received 24 June 2016; published 27 October 2016)

\begin{abstract}
A new operating mode has been developed for the Linac Coherent Light Source (LCLS) in which we shape the longitudinal phase space of the electron beam. This mode of operation is realized using a horizontal collimator located in the middle of the first bunch compressor to truncate the head and tail of the beam. With this method, the electron beam longitudinal phase space and current profile are reshaped, and improvement in lasing performance can be realized. We present experimental studies at the LCLS of the beam shaping effects on the free-electron laser performance.
\end{abstract}

DOI: 10.1103/PhysRevAccelBeams.19.100703

\section{INTRODUCTION}

Over the past several years, great progress has been made in the realization of high power $\mathrm{x}$-ray free-electron lasers (FELs). This revolutionary light source, with $8-10$ orders increase in peak brightness and 2-3 orders decrease in pulse length compared to the pulses from storage ringbased third generation light source, provides an unique tool for ultrafast $\mathrm{x}$-ray studies with atomic spatial resolution $[1,2]$. Nevertheless, there is a continual desire for $\mathrm{x}$-ray pulses with further improved brightness and peak power, driven by $\mathrm{x}$-ray users with applications such as singlemolecule imaging [3] and nonlinear $x$-ray sciences [4]. In a linac-driven FEL, the FEL lasing performance mainly depends on the electron beam brightness, where a combination of low transverse emittance, high peak current and small energy spread are desired.

The final electron beam time-sliced emittance is mostly determined in the photoinjector located at the beginning stage of the linac. The energy spread at the Linac Coherent Light Source (LCLS) is controlled by a laser heater $[5,6]$, which is designed to suppress the microbunching instability and yet achieve a small final slice energy spread. This laser heater "knob" can be easily adjusted during operation for maximizing the FEL photon output. The beam peak current, enhanced by longitudinal compression, is one complicated parameter for optimization since collective effects are involved during the bunch compression, and the nonuniform current profile after compression also causes additional beam brightness degradation in the downstream beamline.

\footnotetext{
*ding@slac.stanford.edu
}

Published by the American Physical Society under the terms of the Creative Commons Attribution 3.0 License. Further distribution of this work must maintain attribution to the author(s) and the published article's title, journal citation, and DOI.
In the linac section, the bunch is compressed in a series of magnetic chicanes in order to achieve a high peak current. To do this, the electron beam is accelerated at an off-crest radio-frequency (rf) phase so that the bunch tail has higher energy than the head. While passing through a four-dipole chicane, the tail travels a shorter path than the head, thus catching up to the head and compressing the bunch in time. The nonlinearities induced by the acceleration and compression processes (e.g., by the longitudinal wakefields, rf curvature, and second order momentum compaction) need to be minimized to avoid high peak current spikes in the temporal distributions [7]. In addition, collective effects such as coherent synchrotron radiation (CSR) in the dipoles can cause significant timedependent energy loss and projected emittance growth [8]. The nonlinearities and the CSR effect are major issues that limit the peak currents that can be achieved.

In this paper, we report on a recent study at the LCLS for improving the FEL performance with a beam shaping technique, which is realized by truncating the double-horn current spikes (at the beam head and tail) with a collimator located at the middle of a bunch compressor. Experimental results have demonstrated that collimation is a robust method for manipulating the current profile and final longitudinal phase space. A flat-top-like current profile with reduced current spikes has been achieved, one that leads to better FEL performance with improved pulse energy, peak power and bandwidth control. In Sec. II, the collimation method and how it helps shape the current profile will be discussed. We then show experimental measurements on the improvement of the FEL pulse energy and peak power in Sec. III, and bandwidth and chirp control in Sec. IV. A discussion and summary are given in Sec. V.

\section{METHODS}

The longitudinal collimation method presented here relies upon the fact that a time-energy chirped beam is 
tilted at a large angle relative to the longitudinal axis in a dispersive section. For example, at the middle of a magnetic chicane where the maximum tilt exists, cutting the beam edges horizontally with a transverse collimator is effectively truncating the bunch head and tail. This correlation-based beam selection has also been adopted in the slotted-foil scheme $[9,10]$. The main difference is that in the slotted foil scheme, although only a fraction of the bunch passing through the slot will lase, the other parts of the bunch remain with the beam but with a spoiled emittance. In the collimation mode here, the electrons hitting the collimator jaws are scraped away, thus the bunch charge in the downstream beam line is reduced.

The longitudinal collimation mode was also studied as a beam slice diagnostic at the Fermi FEL [11] and the LCLS [12]. As discussed in [11], this also has the potential to generate shorter $\mathrm{x}$-ray pulses by truncating most of the charge, with only a small fraction of the bunch passing through the collimator gap. Compared with a low-charge operation mode [13], the collimated beam will have a larger emittance since it starts with a higher charge in the electron gun. However, this collimation mode provides a simple way to shape the electron beam's phase space by truncating a small fraction of the charge at the bunch head and tail [12]. For the beam shaping purpose as studied in this paper, we still keep most of the bunch charge and scrape away a small number of the unwanted particles.

At the LCLS, both the first and second bunch compressor (BC1 and BC2 in Fig. 1) have a horizontal collimator at the middle of the chicane. Considering radiation protection issues, we choose the low-energy chicane $\mathrm{BC} 1$, where the regular operating energy is $220 \mathrm{MeV}$, for collimation. The machine layout is shown in Fig. 1. The collimator and chicane parameters have been discussed in [12]. For example, at the $\mathrm{BC} 1$ collimator location, the horizontal dispersion is $0.23 \mathrm{~m}$, and the rms energy spread is typically about $1 \%$. Thus the horizontal full width of a dispersed beam at the collimator is about $11 \mathrm{~mm}$. Under typical collimating conditions during operation, the collimator gap at the LCLS is set to a full width of 5-7 $\mathrm{mm}$, from which the bunch charge is truncated from initial $250 \mathrm{pC}$ to about $180 \mathrm{pC}$. At the bottom of Fig. 1, we include measured examples of the beam on an optical transition radiation (OTR) screen in the middle of $\mathrm{BC} 1$, without collimation and with collimation; and an example of the final beam's longitudinal distribution measured at the end of the undulator with the collimation mode.

One concern with this collimation mode is the beam quality degradation due to the collective interaction with the collimator jaws. For example, how do the parasitic effects such as the collimator wakefields and longitudinal space charge affect the beam emittance? This has been experimentally investigated earlier and reported on in [12]. It was concluded that the emittance, in our parameter range, is very well preserved after the truncation. Therefore, in this report we leave this concern out and focus on the shaping optimization of the longitudinal phase space for improving the FEL performance.

At the LCLS, a fourth-harmonic rf structure is adopted to linearize the longitudinal phase space before the beam is compressed in $\mathrm{BC} 1$. With the help of this harmonic linearizer, the beam current profile after $\mathrm{BC} 1$ becomes similar to a parabolic distribution (without collimation). In the L2-linac section that follows, the strong longitudinal wakefield in the accelerating structures causes the extreme head and tail portions of the bunch to have a slightly increased slope (3rd-order curvature) in time-energy space. These head and tail parts of the beam are then overcompressed by the $\mathrm{BC} 2$ chicane, leading to large current spikes in the current profile [7]. In Fig. 2 we show simulated examples of phase space and current distributions along the linac in the normal machine configuration (without

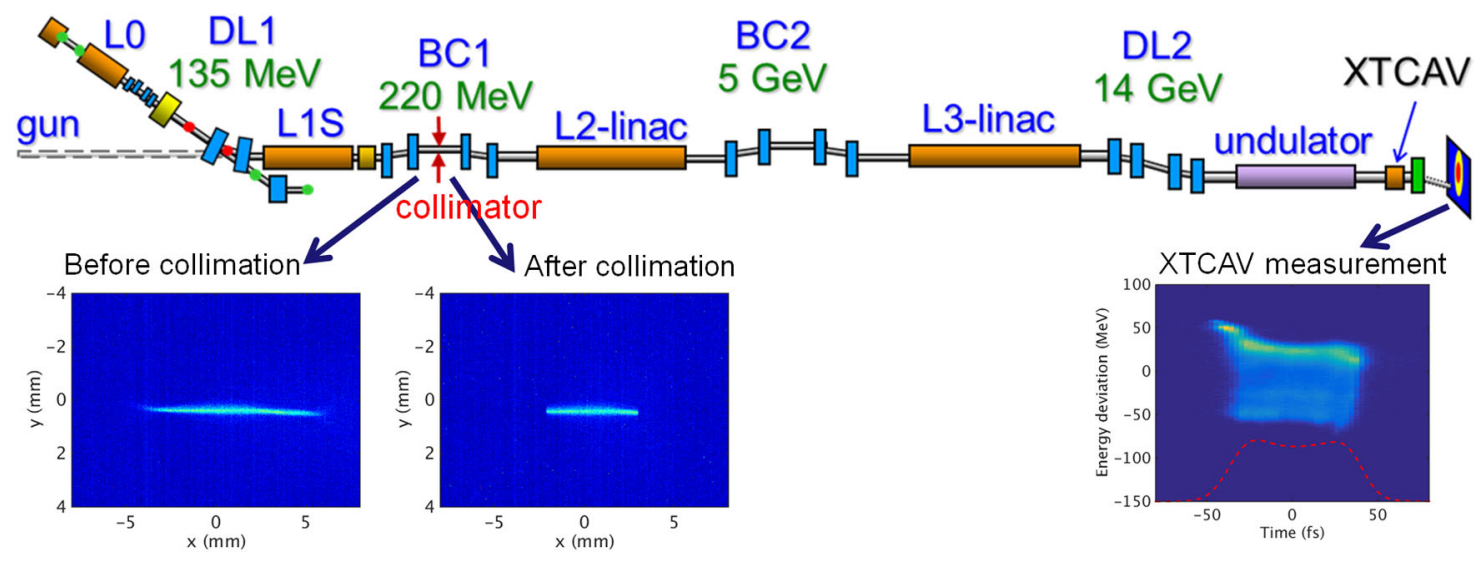

FIG. 1. A schematic of the LCLS machine layout including a collimator in the middle of the first bunch compressor (BC1) and the XTCAV downstream of the FEL undulator. The L2 and L3 are S-band rf linac sections. We also show the measured images at the middle of the $\mathrm{BC} 1$, before and after collimation, and a measured example from XTCAV for the collimated beam after FEL lasing. 

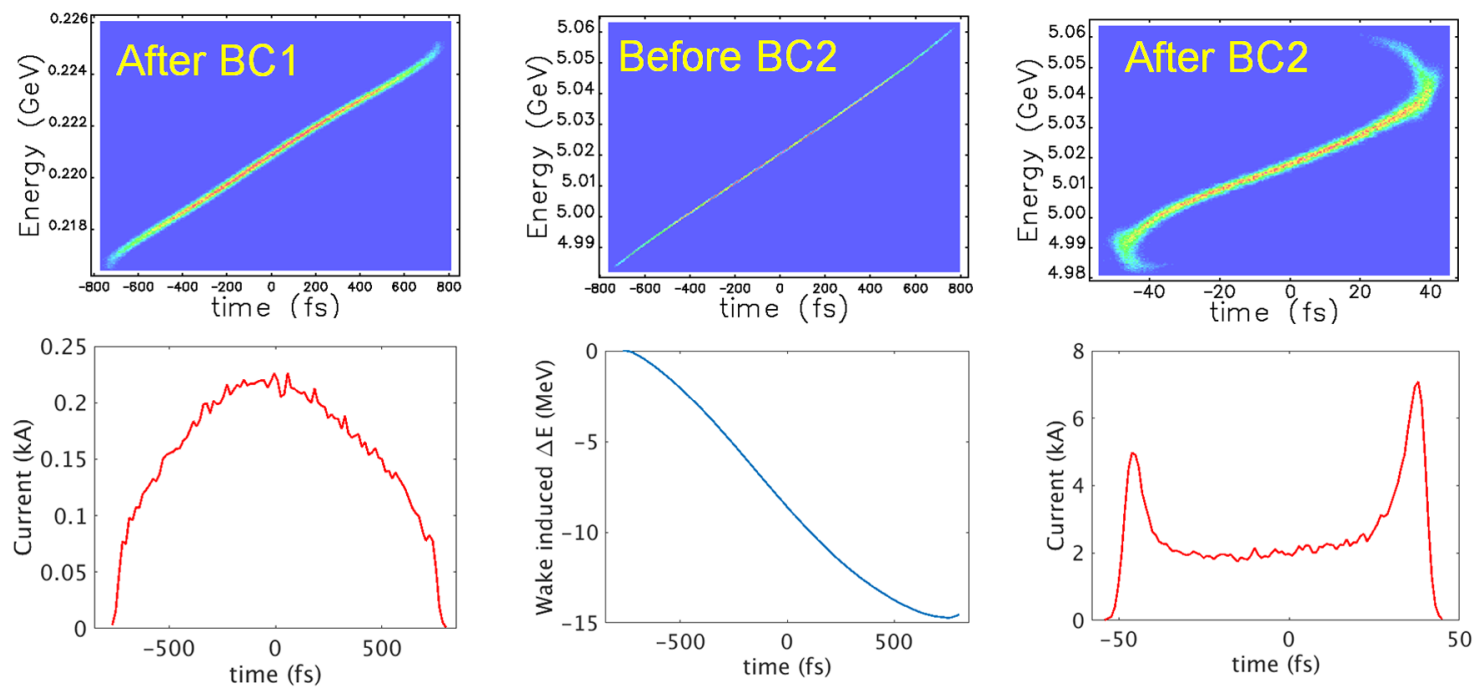

FIG. 2. Simulation examples of a regular beam without collimation, bunch charge $250 \mathrm{pC}$. Left column: electron beam longitudinal phase space and current profile after BC1 chicane. Middle column: longitudinal phase space before BC2 chicane and L2-linac wake induced energy change. Right column: longitudinal phase space and current profile after BC2 chicane.

applying the collimation), where we can see that the energy loss along the bunch has a nonlinear curvature, which leads to a "double-horn" current profile after BC2 compression.

Since the "double-horn" shape shows up during BC2 compression, a natural way to truncate the horns is to use the collimator at the middle of $\mathrm{BC} 2$. In simulations this should work. However, with beam energy typically at $5 \mathrm{GeV}$ in $\mathrm{BC} 2$, the radiation power on the collimator jaws will be much higher than that in the $\mathrm{BC} 1$ region (energy of $220 \mathrm{MeV}$ ). In the following, we explain how the collimation at the $\mathrm{BC} 1$ location also helps shape the final current profile and phase space.

As discussed earlier, L2-linac wakefield induced 3rdorder time-energy curvature is the main source leading to head/tail current spikes. The induced energy loss per unit length from the wakefields is given by a convolution of the point charge wake function and the bunch distribution. We can write down the bunch wake $\mathcal{W}(s)$ - the voltage gain for a test particle at position $s$-as (see, e.g., Ref. [14])

$$
\mathcal{W}(s)=-\int_{0}^{\infty} W\left(s^{\prime}\right) \lambda\left(s-s^{\prime}\right) d s^{\prime},
$$

where $W(s)$ is the rf structure point charge wake function, and $\lambda(s)$ is the longitudinal bunch distribution normalized to 1 . For the SLAC S-band rf structure, the average iris radius $a=11.6 \mathrm{~mm}$, with a typical bunch length in L2linac $\sigma_{z}=0.1 \mathrm{~mm}$, the transient regime of the wake will last $z \sim a^{2} / 2 \sigma_{z}=0.7 \mathrm{~m}$. Since the L2-linac length $L=329 \mathrm{~m}$, much longer than the transient regime, we can use the limiting value of the steady-state wake for this periodic structures [14]:

$$
W\left(0^{+}\right)=\frac{Z_{0} c}{\pi a^{2}},
$$

here $Z_{0}=377 \Omega$, and $c$ is the speed of light in vacuum.

Inserting Eq. (2) to Eq. (1), we obtain the energy change due to the L2-Linac wakefields:

$$
\Delta E(s)=-e^{2} N L \frac{Z_{0} c}{\pi a^{2}} \int_{0}^{\infty} \lambda\left(s-s^{\prime}\right) d s^{\prime} .
$$

Here $N$ is the total number of electrons in the bunch. We emphasize in Eq. (3) that the wake-induced energy loss along the bunch is proportional to an integral of the bunch distribution. For example, a flat-top current profile will cause a linear-variation of the energy loss along the bunch (with more loss in the bunch tail, but no high-order variations). Thus, shaping the current profile from Gaussian/parabolic distribution to a flat-top-like shape will help reduce the 3rd-order curvature in the time-energy correlation due to wakefields. We illustrate this correction with simulation results in Fig. 3. When applying the BC1 collimator, the truncated current profile has a sharp rise in the head and fall in the tail (left column of Fig. 3). After convolving with the wake function, we can see that the wake induced energy loss along the bunch is close to linear (middle column of Fig. 3). Comparing to the case of Fig. 2 we clearly see the improvement in terms of linearity. This linearized energy loss from the L2-linac wakefield leads to much smaller current spikes at the bunch head and tail after BC2 compression. The simulation results of Fig. 3 show the feasibility of linearizing the time-energy correlation by truncating the beam in the low-energy $\mathrm{BC} 1$ region, instead 

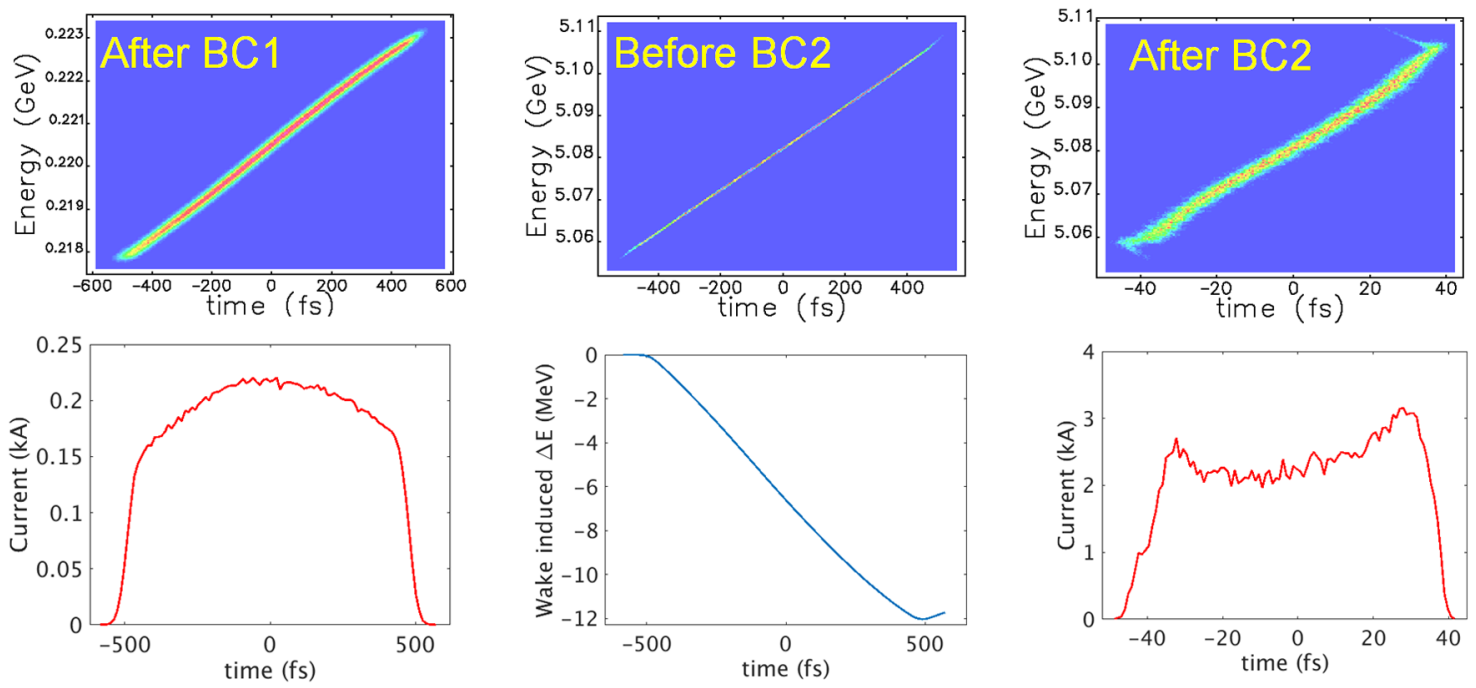

FIG. 3. Simulation examples of the collimated beam. BC1 collimator is used to truncate the bunch charge from $250 \mathrm{pC}$ to $180 \mathrm{pC}$. Left column: electron beam longitudinal phase space and current profile after $\mathrm{BC} 1$ chicane. Middle column: longitudinal phase space before BC2 chicane and L2-linac wake induced energy change. Right column: longitudinal phase space and current profile after BC2 chicane.

of in the high-energy $\mathrm{BC} 2$ region, which greatly reduces the radiation concerns of beam hitting on the collimator jaws.

\section{BEAM SHAPING AND FEL LASING IMPROVEMENT}

The LCLS undulator line is based on fixed gap permanent magnet undulators. A canted pole design allows small adjustments of the undulator strength $K$. This feature is used for post-saturation taper [1], two-color pulses [15,16], and reverse taper schemes [17]. Large photon energy adjustments are made by changing the electron beam energy. The present deliverable x-ray photon energy covers both soft and hard x-ray spectra, ranging from $250 \mathrm{eV}$ to $12 \mathrm{keV}$ [18]. This requires the electron beam energy to vary from $2.4 \mathrm{GeV}$ to $16 \mathrm{GeV}$. The peak current requirement for soft and hard x-rays is also different, typically over $3 \mathrm{kA}$ for hard x-ray FELs, while 1-2 kA for soft x-ray FELs. We use a hard x-ray FEL example (photon energy of $7 \mathrm{keV}$ ) to discuss the lasing performance with a collimated beam in what follows.

For an LCLS FEL at $7 \mathrm{keV}$, the electron beam energy is $\sim 12.5 \mathrm{GeV}$. We list the major machine parameters used during the measurement in Table I. The charge is truncated by the $\mathrm{BC} 1$ collimator from $250 \mathrm{pC}$ to $180 \mathrm{pC}$. An X-band transverse deflector (XTCAV) [19], located downstream of the undulator section, provides a direct diagnostic for this beam shaping study. This deflector includes two 1-m-long $\mathrm{X}$-band rf deflecting structures, providing a time-dependent horizontal kick on the beam. It is followed by a vertically bending spectrometer magnet, and the beam is imaged on a downstream yttrium aluminium garnet (YAG) screen. With this arrangement, the horizontal dimension of the measured image represents time while the vertical dimension represents energy. The horizontal scale of the image can be calibrated in absolute units of time by measuring the beam centroid position as a function of rf phase in degrees of $\mathrm{X}$-band at the deflector. Similarly, the vertical scale is calibrated with beam energy shifts. Thus, the XTCAV system provides a direct measurement of the electron beam time-energy phase space. In addition, since the location of the measurement is downstream of the FEL lasing process, the lasing induced time-resolved energy loss or energy spread growth can also be measured. By comparing with lasing-off images (suppress the lasing with an oscillating orbit inside the undulator beam line) one can reconstruct the FEL power profile [20].

Figure 4(a) and (b) show measured examples of the collimated beam longitudinal phase space with lasing-off and lasing-on conditions. The electron beam current profile and reconstructed $\mathrm{x}$-ray power profile are shown in (c). The XTCAV was operated at $65 \mathrm{MV}$ during the measurement with a recently developed compact X-band SLED mode [21], providing a time resolution of $2.5 \mathrm{fs} r \mathrm{rms}$ for the beam energy of $12.5 \mathrm{GeV}$. We see in this example, the peak

TABLE I. Main machine parameters for Fig. 4 at $7 \mathrm{keV}$.

\begin{tabular}{lccc}
\hline \hline Parameter & Symbol & Value & Unit \\
\hline Bunch charge at injector & $Q_{0}$ & 250 & $\mathrm{pC}$ \\
Bunch charge after BC1 & $Q$ & 180 & $\mathrm{pC}$ \\
Beam energy at BC1 & $E_{1}$ & 220 & $\mathrm{MeV}$ \\
Beam energy at BC2 & $E_{2}$ & 5 & $\mathrm{GeV}$ \\
Beam final energy & $E_{f}$ & 12.5 & $\mathrm{GeV}$ \\
BC1 current & $I_{1}$ & 220 & $\mathrm{~A}$ \\
BC2 current & $I_{2}$ & $3-5$ & $\mathrm{kA}$ \\
BC1 collimator gap & $g$ & $\sim 6$ & $\mathrm{~mm}$ \\
BC1 R56 & & -45.5 & $\mathrm{~mm}$ \\
BC2 R56 & & -28 & $\mathrm{~mm}$ \\
\hline \hline
\end{tabular}



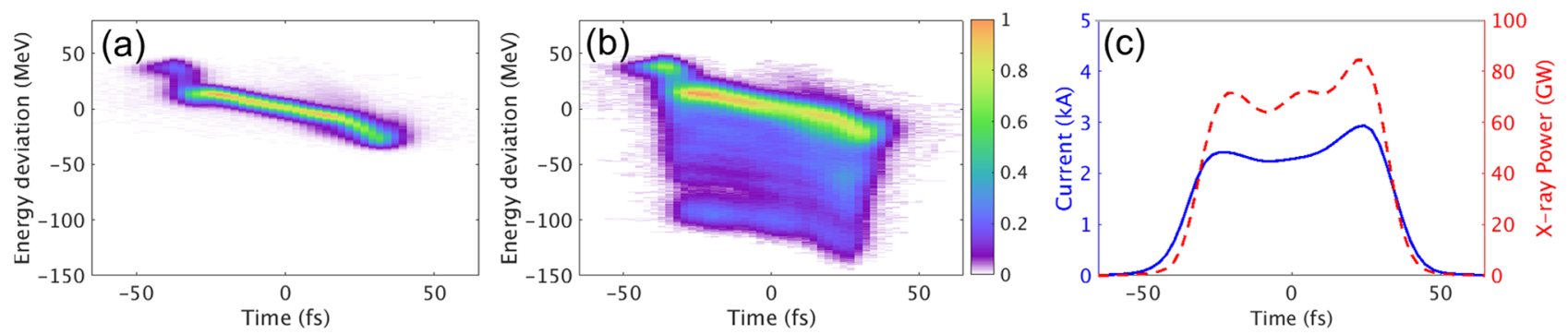

FIG. 4. Measurement examples of the collimated beam for hard x-ray FEL case with photon energy 7 keV. The electron beam energy is 12.5 GeV, and other machine parameters can be found in Table I. The electron beam longitudinal phase space is shown in (a) for lasingoff condition and (b) for lasing-on condition; in (c) the beam current profile and reconstructed FEL power profile are given. In this example the x-ray pulse energy is $4.8 \mathrm{~mJ}$. Data taken on 3/15/2016.

current is about $2.5 \mathrm{kA}$ with a relatively uniform profile, which agrees well with the simulation predictions of Fig. 3. Since the current profile and the longitudinal phase space are getting uniform, we can see that a uniform lasing along the bunch can be achieved, producing a pulse energy over $4 \mathrm{~mJ}$ in a routine operation at this energy. The x-ray peak power ranges from 50 to $100 \mathrm{GW}$ depending on the pulse duration.

As a comparison, we show two measurement examples in Fig. 5, where no collimation was applied. These examples were measured in 2013 with bunch charge of $250 \mathrm{pC}$ and $150 \mathrm{pC}$, respectively. The beam energy was $13.5 \mathrm{GeV}$, and the XTCAV was operated at $45 \mathrm{MV}$. This was the maximum achievable deflecting voltage at that time (the SLED mode was not available yet). The XTCAV time resolution was about $4 \mathrm{fs}$ rms. We see a "double-horn" shape current profile shows up in both cases. We remark that the absolute value of the current spike could be underestimated due to the resolution limit. Nevertheless, comparing to the collimated example in Fig. 4, at a similar core current level, here we see more pronounced current variations even with a lower time resolution. The current horns cause strong local energy loss due to CSR, which makes energy variation along the bunch and the lasing nonuniform. In these two examples, the x-ray pulse energy is about $2 \mathrm{~mJ}$, and the power is below $50 \mathrm{GW}$. Since the current spikes are getting stronger when charge increases, to compromise between the photon output and the collective effects, the LCLS typically operates at $150-180 \mathrm{pC}$ bunch charge from the injector for hard $\mathrm{x}$ rays over the past few years, until the recent collimation mode as reported in this paper was developed. With further tuning up, the best measured $\mathrm{x}$-ray pulse energy using 150-180 pC bunch charge (without collimation) was about $3 \mathrm{~mJ}$ (see Fig. 6 in the following).
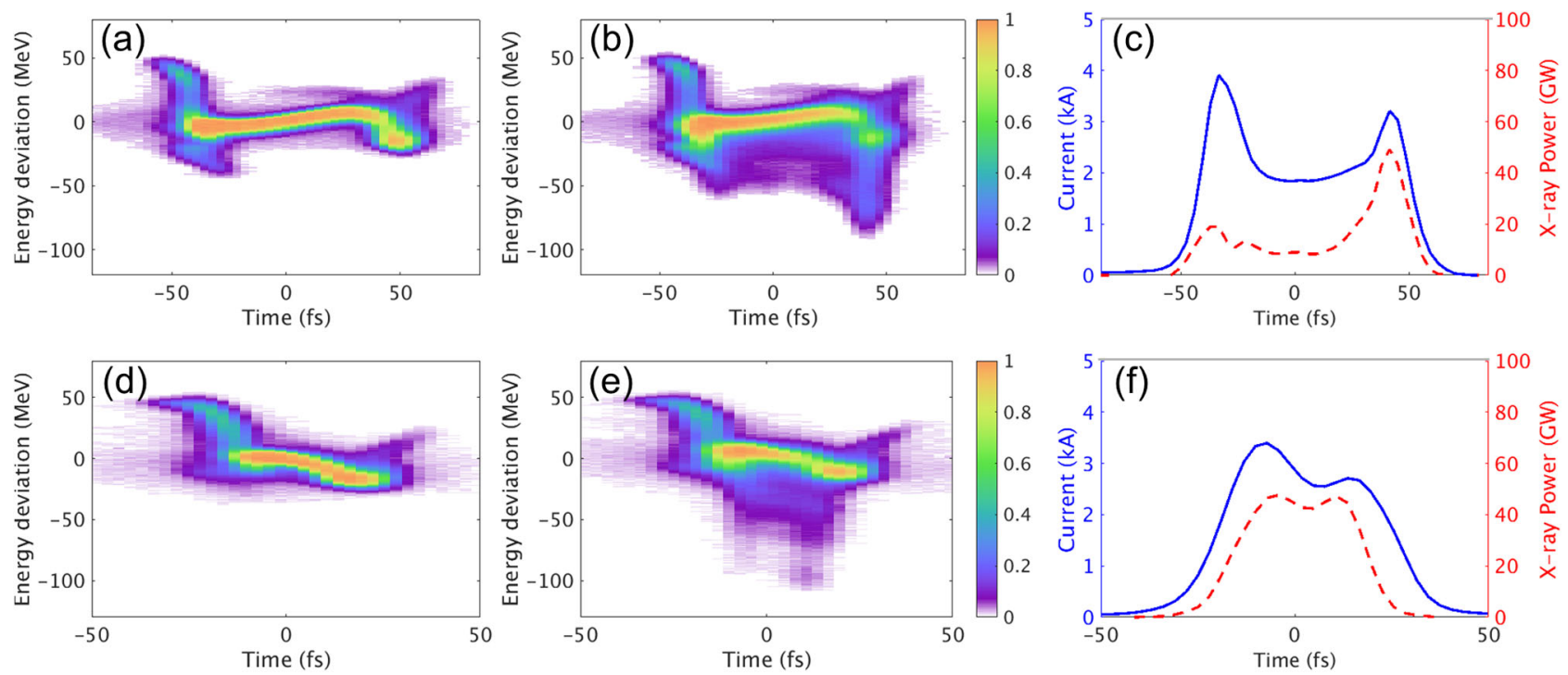

FIG. 5. Measurement examples of the final beam without collimation for hard x-ray FEL case. Electron beam energy is 13.5 GeV, and bunch charge is $250 \mathrm{pC}$ (top row) and $150 \mathrm{pC}$ (bottom row), respectively. (a)-(c): bunch charge $250 \mathrm{pC}$, the electron beam longitudinal phase space for lasing-off condition (a) and lasing-on condition(b), and the beam current profile and reconstructed FEL power profile in (c). (d)-(f): bunch charge $150 \mathrm{pC}$, same measurement as $250 \mathrm{pC}$ case. In both cases the x-ray pulse energy is $\sim 2 \mathrm{~mJ}$. Data taken on $11 /$ $16-17 / 2013$. 


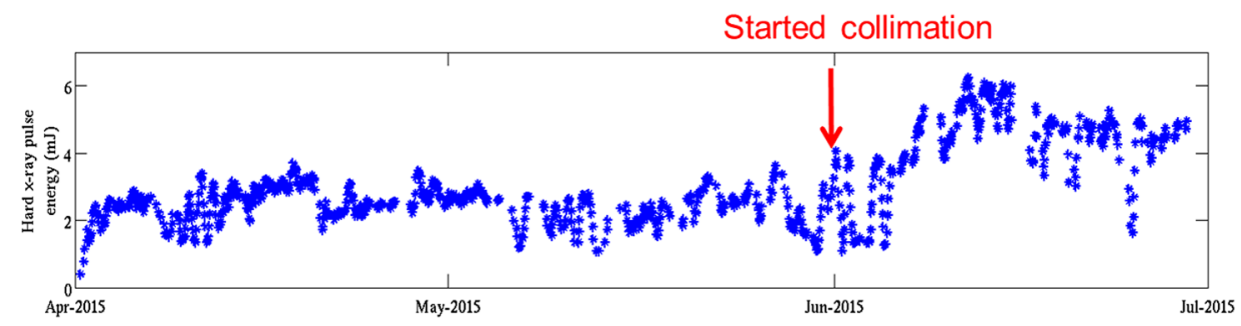

FIG. 6. Archived gas detector history to show the recorded FEL pulse energy before and after using the collimation mode. The photon energy is filtered between 7 to $9 \mathrm{keV}$. The bunch charge for the period before using the collimation mode was $180 \mathrm{pC}$ from the injector to the undulator, while with the collimation mode, the bunch charge was truncated from $250 \mathrm{pC}$ to $180 \mathrm{pC}$ with $\mathrm{BC} 1 \mathrm{collimator}$. The actual bunch charge for FEL lasing in this plot is the same.

In the above examples, we use hard $\mathrm{x}$-ray examples to demonstrate the shaping method. In the soft x-ray regime, the required beam peak current is lower, thus we typically have smaller current horns (measurement examples can be found in [19]). However, the longitudinal space charge force is stronger due to lower electron beam energy, which causes longitudinal phase space distortion even with small current horns. Experimental observations show obvious improvement also in soft x-ray regime after cutting the current horns. As the present LCLS operation, we use the collimation mode as a routine setup for both hard and soft x-ray FEL users.

Comparing the results from collimation mode and noncollimation mode in Fig. 4 and Fig. 5, we see the FEL lasing efficiency is clearly improved. Here we discuss briefly how this shaping helps the FEL lasing amplification. During the beam acceleration and compression, the major collective effects are from longitudinal space charge force, CSR and longitudinal wakefields. CSR and longitudinal wakes cause a time-dependent energy variation along the bunch. Since this energy loss is a convolution between the wake function and the current distribution, with a doublehorn current profile, the CSR and wakefield induced energy variation along the bunch increases [14,22]. After passing through bending magnets, this time-dependent energy loss will cause Twiss parameter variation along the bunch and also projected emittance growth. In the downstream beam line, transverse matching and undulator tapering have to be compromised between these time-dependent variations. Typically this variation leads to a partial (nonuniform) lasing in the bunch as seen in Fig. 5, and also causes radiation wavelength shifts and bandwidth growth. The longitudinal space charge force is proportional to the derivative of the instantaneous current. It vanishes for a uniform beam, but for a beam with current spikes, it always accelerates the electrons in the spike head and decelerates those in the spike tail. All these time-dependent problems are greatly reduced with the reshaped flat-top-like beam, and we see uniform lasing along the bunch in Fig. 4(b). This achieved uniform lasing feature not only helps extract more photons from the electron beam, but also simplifies the machine tuning. A practical FEL tuning optimization may be biased by the lasing in the current horns, which now can be avoided since the entire bunch is lasing uniformly. Note if we want to get a similar final charge after collimation, we have to start with a higher charge in the gun, which could give a slightly larger emittance due to stronger transverse space charge forces. With the LCLS parameters, we measured about $10 \%$ emittance growth when increasing the charge from $180 \mathrm{pC}$ to $250 \mathrm{pC}$. The FEL measurements show that we still win with a collimated beam considering this small emittance growth.

During LCLS operation for x-ray user experiments, unless there is a special requirement on the pulse duration or spectral bandwidth, the machine is typically optimized to achieve maximum pulse energy. The typical adjustments ("knobs") include the injector emittance, final peak current, energy spread, undulator tapering, transverse matching, etc. The setup can vary at different photon energies. In Fig. 6 we show the LCLS performance achieved between April and July 2015 operating in the hard X-ray regime between 7 and $9 \mathrm{keV}$. The experimental data show a clear improvement in the performance after the collimation mode was started at the very end of May 2015. As details of the machine setup are ignored and only the pulse energy measured by the gas detector [23] is shown, it means that collimation provided general benefit to all the operating modes. Before applying the collimation mode, the bunch charge was $180 \mathrm{pC}$ from the injector, and in the hard $\mathrm{x}$-ray spectral range, the maximum pulse energy was about $3.5 \mathrm{~mJ}$. After truncating the bunch head/tail using the collimator (from $250 \mathrm{pC}$ cutting down to $180 \mathrm{pC}$ ), we can see that the FEL intensity ramped up to over $4 \mathrm{~mJ}$ after a few days, and even exceeded $5 \mathrm{~mJ}$ in some conditions.

\section{CHIRP AND BANDWIDTH CONTROL}

\section{A. Electron beam chirp control}

For some x-ray experiments requiring narrow bandwidth in the self-amplified spontaneous emission (SASE) mode, or the self-seeding mode, precise control of the time-energy chirp of the electron beam is important. There are two main reasons for a broadening of the radiation bandwidth. One is the linear chirp in the electron beam time-energy phase 
space. In a FEL facility, a time-energy chirp (lower energy at the bunch head) is necessary for the purpose of longitudinal compression. After the compressor, this energy chirp is designed to be canceled by the downstream linac structure and transport line wakefields. Since this wakefield dechirping effect is related to the beam current distribution, when the bunch length (beam current) has to be varied for different user requirement, the final beam could have a residual chirp that is not fully canceled. Another reason to cause bandwidth widening is the nonlinear energy variation along the bunch, as discussed in the last section. This time-dependent variation gets stronger with a double-horn current structure. Since the SASE radiation is independent over the coherence time (temporal spike) [1], for a typical bunch whose duration is much longer than the coherence time, the FEL radiation wavelength shifts around determined by the local energy variation. This will cause bandwidth growth and reduce the spectral brightness.

For the XTCAV diagnostic, note that the measured longitudinal phase space is downstream of the undulator beam line, which includes the wakefield effect from the undulator vacuum chamber. Since what we care about is to have a flat-chirp beam at the entrance of the undulator, a model-based wakefield subtraction has been developed to reconstruct the initial longitudinal phase space right before entering the undulator.

The wakefield in the undulator chamber comes from different sources, such as the resistive walls, geometry transitions, surface roughness, etc. Earlier studies verified that for the LCLS parameters, the dominant part is the resistive wall wakefield [24]. Thus for our reconstruction, we just subtract the resistive wall induced energy variation.

The point charge resistive wall wake including ac conductivity from the undulator chamber with a flat pipe can be approximated by a simple damped oscillator model [25]:

$$
W(s)=\frac{\pi^{2}}{16} \frac{Z_{0} c}{\pi a^{2}} e^{-k_{r} s / 2 Q_{r}} \cos \left(k_{r} s\right)
$$

where $a$ is the beam pipe radius (half of the gap for the flat pipe case, $2.5 \mathrm{~mm}$ for LCLS), and $k_{r}$ and $Q_{r}$ are fitting parameters as a function of $\Gamma$. Here $\Gamma$ is a dimensionless relaxation time $\Gamma=c \tau / s_{0}$. For the LCLS aluminum chamber, the relaxation time $\tau=8 \mathrm{fs}$, and the characteristic distance $s_{0}=9.8 \mu \mathrm{m} \mathrm{[25] \text {. }}$

Since we have the measured current profile, the energy loss from the undulator chamber resistive wall wake can be calculated by inserting Eq. (4) into Eq. (1), multiplying by the bunch charge and the undulator chamber length $\sim 130 \mathrm{~m}$. By adding back this loss, we can reconstruct the longitudinal phase space before the undulator. An example shown in Fig. 7 further demonstrates the procedure. Three steps are explained in the figure: (1) obtain the current profile by constructing the histogram in time from the measured phase space and normalizing with the recorded bunch charge; (2) calculate the wakefield induced energy loss by convolving the point charge wake with the current profile; (3) add back the energy loss to reconstruct the beam initial longitudinal phase space at the entrance of the undulator.

The example in Fig. 7 shows the reconstructed longitudinal phase space at the entrance of the undulator with a pretty flat chirp, which is important for achieving narrowbandwidth FELs. In a FEL facility with two-stage compressors, the final energy chirp can be adjusted by changing the compression ratio between the two compressors. We use two measurement examples in Fig. 8 to explain this effect. The two bunches here show the reconstructed phase space at the entrance of the undulator, with beam energy of
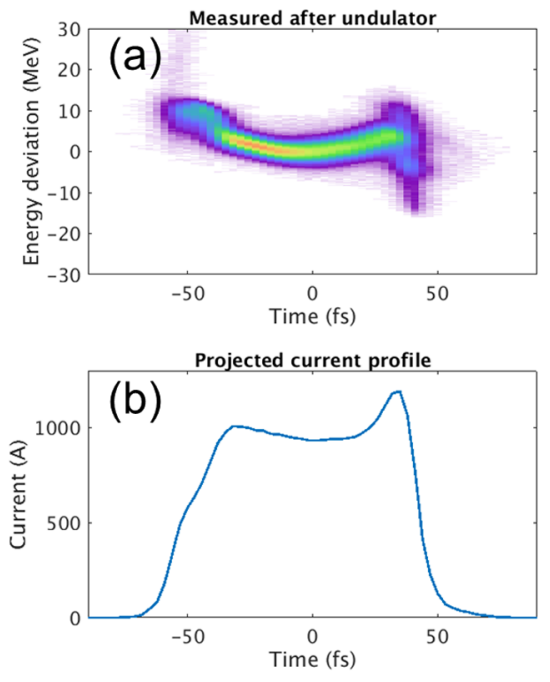
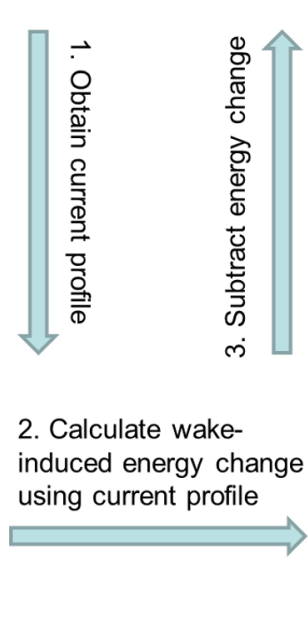
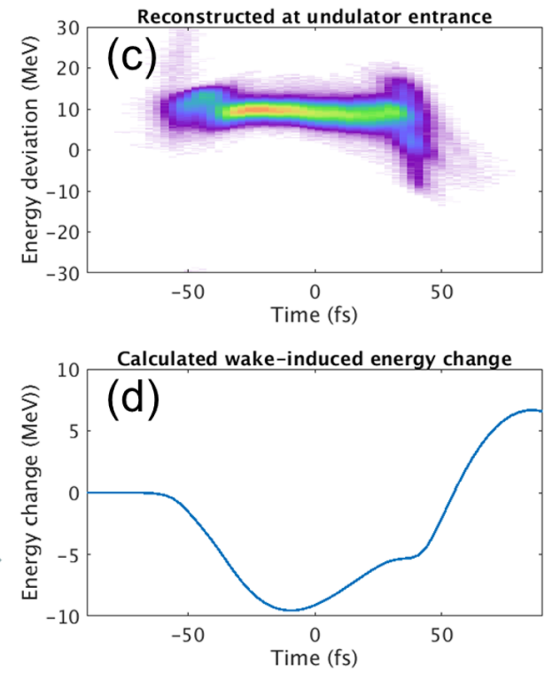

FIG. 7. The procedure to retrieve the initial phase space at the undulator entrance. The final measured longitudinal phase space is in (a), and by projecting to time, we obtain the current profile (b). Using the resistive wall wakefield model, the induced energy loss is calculated in (d). Finally the initial phase space (c) is obtained by subtracting the energy loss in (d) from (a). Bunch head is to the left. 

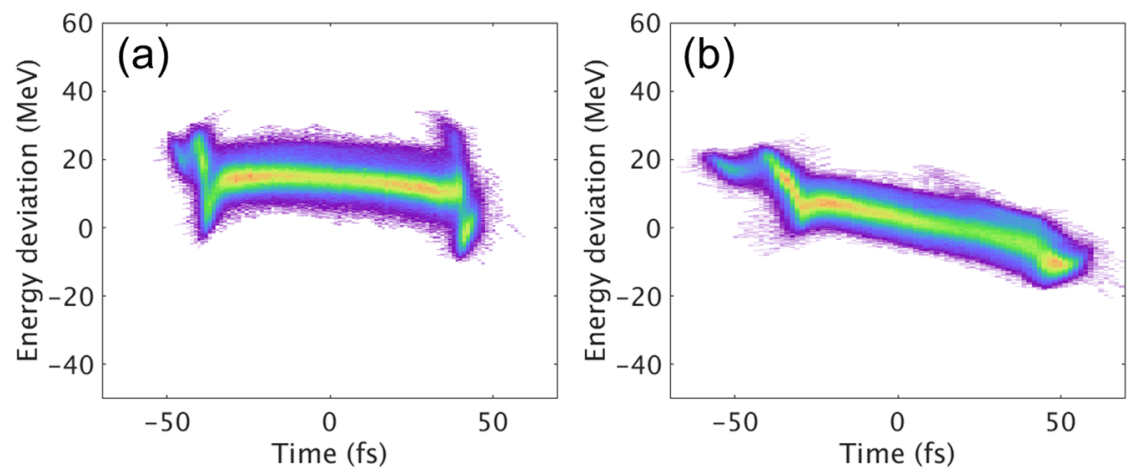

FIG. 8. The final phase space of the collimated beam at the entrance of the undulator with different setting of the beam current after BC1: (a) $130 \mathrm{~A}$; (b) $180 \mathrm{~A}$. The final beam energy at the undulator entrance is $4 \mathrm{GeV}$, with current about $1500 \mathrm{~A}$. The bunch head is to the left.

$4 \mathrm{GeV}$, and same final current $\sim 1500 \mathrm{~A}$. In the case (a), it is a nominal setup with the current after $\mathrm{BC} 1$ of $130 \mathrm{~A}$; while in case (b), we increase the $\mathrm{BC} 1$ compression and the current after $\mathrm{BC} 1$ is $180 \mathrm{~A}$. To get a same final current after $\mathrm{BC} 2$ compression, here for the case (b), we have to reduce the compression in $\mathrm{BC} 2$ using a smaller chirp. Since the downstream L3 structure wakefields will dechirp the bunch by a similar amount at the same current, the smaller initial chirp after BC2 in case (b) will be overcanceled, and we see the bunch head has higher energy than the tail (a negative chirp). Thus, for a same final current after $\mathrm{BC} 2$, we can adjust the compression ratio between the two compressors to balance the final beam chirp. Too much compression in BC1 will cause a negative chirp (residual chirp is overcanceled by L3 wakefields). It is also necessary sometimes to adjust the collimator center for further optimization of the current profile symmetry and chirp.

\section{B. FEL spectral measurement}

At the LCLS, both hard and soft x-ray spectra can be measured with two different single-shot spectrometers
$[26,27]$. We use hard x-ray examples to demonstrate the spectral improvement with the collimated beam. The electron beam chirp control using XTCAV is the first step to set up the machine, then we use the spectrometer as the ultimate diagnostic to confirm a minimum chirp by measuring the SASE FEL bandwidth. In Fig. 9, we show the measured FEL spectra without and with collimator: (a) full bunch charge of $150 \mathrm{pC}$ without collimation, at $7 \mathrm{keV}$; (b) bunch charge same as (a) but at $9.3 \mathrm{keV}$; and (c) collimation mode cutting from $250 \mathrm{pC}$ to $180 \mathrm{pC}$ at $7.9 \mathrm{keV}$. In these examples, the setup was optimized for minimum bandwidth, and used post-saturation taper for achieving maximum pulse intensity. The pulse energy is about $2.7 \mathrm{~mJ}, 2.6 \mathrm{~mJ}$, and $4.3 \mathrm{~mJ}$, respectively. The averaged spectrum (after filtering out the electron beam energy jitter) in the collimated case shows a Gaussian distribution, while the noncollimated cases usually shows an asymmetric distribution with an energy tail. We can see that the averaged bandwidth is reduced from about $25 \mathrm{eV}$ to $15 \mathrm{eV}$ after applying collimation, and the spectral peak intensity doubles. As discussed earlier, for the noncollimated case, the electron beam energy near the double-horn

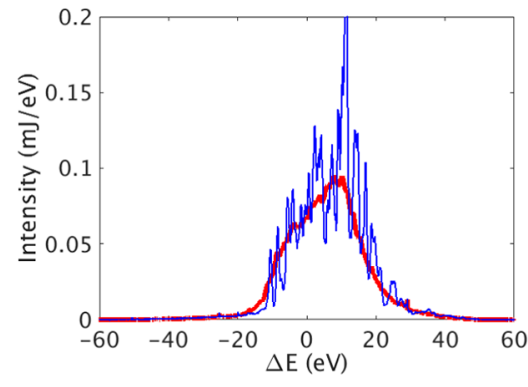

(a) $150 \mathrm{pC}$, without collimation, $7 \mathrm{keV}$

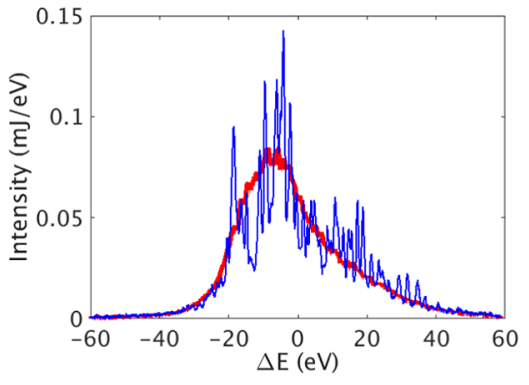

(b) $150 \mathrm{pC}$, without collimation, $9.3 \mathrm{keV}$

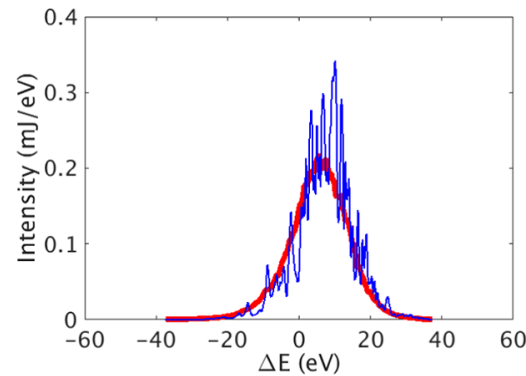

(c)Collimation mode, $180 \mathrm{pC}, 7.9 \mathrm{keV}$

FIG. 9. The measured hard x-ray SASE FEL spectra for operating mode without collimation (a) and (b), and with collimation (c). The blue curve is a single-shot example, and the thick red curve is averaged with 100 shots. In (a), measured on 5/23/15, the bunch charge is $150 \mathrm{pC}$ from injector to the undulator, photon energy is $7 \mathrm{keV}$, bandwidth is $\sim 25 \mathrm{eV} \mathrm{FWHM}$, and pulse energy is about $2.7 \mathrm{~mJ}$; in (b), measured on $4 / 29 / 15$, the bunch charge is $150 \mathrm{pC}$ from injector to the undulator, photon energy is $9.3 \mathrm{keV}$, bandwidth is $\sim 25 \mathrm{eV}$ FWHM, and the pulse energy is $2.6 \mathrm{~mJ}$; in (c), measured on 6/23/16, the charge is truncated with $\mathrm{BC} 1 \mathrm{collimator}$ from $250 \mathrm{pC}$ to $180 \mathrm{pC}$, photon energy is $7.9 \mathrm{keV}$, bandwidth is $\sim 15 \mathrm{eV}$, and the pulse energy is $4.3 \mathrm{~mJ}$. 
area typically has a variation due to CSR. If lasing happens in this area, this energy variation could cause a wider bandwidth or additional tails in the spectral distribution. Note if we kick the beam at the saturation point, the bandwidth can be further reduced, with a sacrifice of photons.

A uniform beam shape also helps for self-seeding operation, as developed at the LCLS in both hard x-ray $[28,29]$ and soft $x$-ray [30] regime. After generating a narrow bandwidth seed from the monochromator, this seed would be amplified in the downstream undulators. Any distortion on the beam phase space distribution or a residual time-energy chirp will cause spectral broadening in the selfseeded radiation pulse, and degrade the peak spectral brightness [31,32]. The beam shaping discussed here provides a better control of the longitudinal phase space, which helps the quality of the seeded FEL output. One recent study with the collimated beam for hard x-ray selfseeding can be found in [33], and further studies will follow with more self-seeding operation experience.

\section{SUMMARY}

We have developed a new operating mode with longitudinal beam shaping for improving the FEL performance at the LCLS. This is based on the existing hardware and can be easily applied to other accelerator facilities using normal conducting rf. With a more uniform current distribution after truncating the head/tail horns, some collective effects such as CSR, space charge and wakefields are reduced. This greatly helps transverse matching and FEL optimization. An increase of the FEL output and peak power with a better controlled spectral bandwidth has been achieved at the LCLS. This shaping method has been adopted at the LCLS as a routine operation mode for both SASE and selfseeding FEL operations.

\section{ACKNOWLEDGMENTS}

We thank SLAC operations and engineering groups for their dedicated support. This work was supported by Department of Energy Contract No. DE-AC0276 SF00515.

[1] Z. Huang and K.-J. Kim, Review of x-ray free-electron laser theory, Phys. Rev. ST Accel. Beams 10, 034801 (2007).

[2] B. W. J. McNeil and N. R. Thompson, X-ray free-electron lasers, Nat. Photonics 4, 814 (2010).

[3] A. Aquila et al., The linac coherent light source single particle imaging road map, Structural Dynamics 2, 041701 (2015).

[4] R. W. Schoenlei et al., New science opportunities enabled by LCLS-II x-ray lasers, SLAC Report No. SLAC-R-1053, 2015.
[5] Z. Huang, M. Borland, P. Emma, J. Wu, C. Limborg, G. Stupakov, and J. Welch, Suppression of microbunching instability in the linac coherent light source, Phys. Rev. ST Accel. Beams 7, 074401 (2004).

[6] Z. Huang, A. Brachmann, F.-J. Decker, Y. Ding, D. Dowell, P. Emma, J. Frisch, S. Gilevich, G. Hays, P. Hering, R. Iverson, H. Loos, A. Miahnahri, H.-D. Nuhn, D. Ratner, G. Stupakov, J. Turner, J. Welch, W. White, J. $\mathrm{Wu}$, and D. Xiang, Measurements of the linac coherent light source laser heater and its impact on the X-ray freeelectron laser performance, Phys. Rev. ST Accel. Beams 13, 020703 (2010).

[7] J. Arthur et al., Linac Coherent Light Source (LCLS) Conceptual Design Report No. SLAC-R-593, 2002.

[8] K. L. F. Bane, F.-J. Decker, Y. Ding, D. Dowell, P. Emma, J. Frisch, Z. Huang, R. Iverson, C. Limborg-Deprey, H. Loos, H.-D. Nuhn, D. Ratner, G. Stupakov, J. Turner, J. Welch, and J. Wu, Measurements and modeling of coherent synchrotron radiation and its impact on the linac coherent light source electron beam, Phys. Rev. ST Accel. Beams 12, 030704 (2009).

[9] P. Emma, K. Bane, M. Cornacchia, Z. Huang, H. Schlarb, G. Stupakov, and D. Walz, Femtosecond and Subfemtosecond X-ray Pulses from a Self-Amplified SpontaneousEmissionbased Free-Electron Laser, Phys. Rev. Lett. 92, 074801 (2004).

[10] Y. Ding, C. Behrens, R. Coffee, F.-J. Decker, P. Emma, C. Field, W. Helml, Z. Huang, P. Krejcik, J. Krzywinski, H. Loos, A. Lutman, A. Marinelli, T. J. Maxwell, and J. Turner, Generating femtosecond x-ray pulses using an emittance-spoiling foil in free-electron lasers, Appl. Phys. Lett. 107, 191104 (2015).

[11] S. Di Mitri, D. Castronovo, I. Cudin, and L. Fröhlich, Electron slicing for the generation of tunable femtosecond soft x-ray pulses from a free electron laser and slice diagnostics, Phys. Rev. ST Accel. Beams 16, 042801 (2013).

[12] F. Zhou, K. Bane, Y. Ding, Z. Huang, H. Loos, and T. Raubenheimer, Measurements and analysis of a highbrightness electron beam collimated in a magnetic bunch compressor, Phys. Rev. ST Accel. Beams 18, 050702 (2015).

[13] Y. Ding, A. Brachmann, F.-J. Decker, D. Dowell, P. Emma, J. Frisch, S. Gilevich, G. Hays, P. Hering, Z. Huang et al., Measurements and Simulations of Ultralow Emittance and Ultrashort Electron Beams in the Linac Coherent Light Source, Phys. Rev. Lett. 102, 254801 (2009).

[14] K. L. F. Bane, Wakefields of sub-picosecond electron bunches, Report No. SLAC-PUB-11829, 2006.

[15] A. A. Lutman, R. Coffee, Y. Ding, Z. Huang, J. Krzywinski, T. Maxwell, M. Messerschmidt, and H.-D. Nuhn, Experimental Demonstration of Femtosecond TwoColor X-ray Free-Electron Lasers, Phys. Rev. Lett. 110, 134801 (2013).

[16] A. Marinelli, A. A. Lutman, J. Wu, Y. Ding, J. Krzywinski, H.-D. Nuhn, Y. Feng, R. N. Coffee, and C. Pellegrini, Multicolor Operation and Spectral Control in a GainModulated X-ray Free-Electron Laser, Phys. Rev. Lett. 111, 134801 (2013).

[17] A. A. Lutman, J. P. MacArthur, M. Ilchen, A. O. Lindahl, J. Buck, R. N. Coffee, G. L. Dakovski, L. Dammann, Y. 
Ding, H. A. Dürr et al., Polarization control in an x-ray free-electron laser, Nat. Photonics 10, 468 (2016).

[18] P. Emma, R. Akre, J. Arthur, R. Bionta, C. Bostedt, J. Bozek, A. Brachmann, P. Bucksbaum, R. Coffee, F.-J. Decker et al., First lasing and operation of ånangstromwavelength free-electron laser, Nat. Photonics 4, 641 (2010).

[19] C. Behrens, F.-J. Decker, Y. Ding, V. Dolgashev, J. Frisch, Z. Huang, P. Krejcik, H. Loos, A. Lutman, T. Maxwell et al., Few-femtosecond time-resolved measurements of X-ray free-electron lasers, Nat. Commun. 5, 3762 (2014).

[20] Y. Ding, C. Behrens, P. Emma, J. Frisch, Z. Huang, H. Loos, P. Krejcik, and M.-H. Wang, Femtosecond x-ray pulse temporal characterization in free-electron lasers using a transverse deflector, Phys. Rev. ST Accel. Beams 14, 120701 (2011).

[21] J. Wang et al., R\&D for a super compact sled system at slac, in Proceedings of the 7th International Particle Accelerator Conference, Busan, Korea, 2016 (JACoW, Busan, Korea, 2016), p. MOOCA01.

[22] S. Reiche, W. Fawley, K. Bane, P. Emma, Z. Huang, H.-D. Nuhn, and G. Stupakov, Optimization of the lcls X-ray fel output performance in the presence of strong undulator wakefields, in Proceedings of the 21 st Particle Accelerator Conference, Knoxville, TN, 2005 (IEEE, Piscataway, NJ, 2005), p. 2396.

[23] S. Moeller et al., Photon beamlines and diagnostics at LCLS, Nucl. Instrum. Methods Phys. Res., Sect. A 635, S6 (2011).

[24] A. Novokhatski, Calculating the loss factor of the lcls beam line elements for ultra short bunches, in Proceedings of the 31st International Free Electron Laser Conference (FEL 09), Liverpool, UK (STFC Daresbury Laboratory, Warrington, 2009), p. WEPC22.

[25] K. L. F. Bane, Resistive wall wakefield in the lcls undulator beam pipe, Report No. SLAC-PUB-10707, 2004.

[26] D. Zhu, M. Cammarata, J. M. Feldkamp, D. M. Fritz, J. B. Hastings, S. Lee, H. T. Lemke, A. Robert, J. L. Turner, and Y. Feng, A single-shot transmissive spectrometer for hard
X-ray free electron lasers, Appl. Phys. Lett. 101, 034103 (2012).

[27] P. Heimann, O. Krupin, W. F. Schlotter, J. Turner, J. Krzywinski, F. Sorgenfrei, M. Messerschmidt, D. Bernstein, J. Chalupsk, V. Hjkov, S. Hau-Riege, M. Holmes, L. Juha, N. Kelez, J. Lning, D. Nordlund, M. Fernandez Perea, A. Scherz, R. Soufli, W. Wurth, and M. Rowen, Linac coherent light source soft $\mathrm{x}$-ray materials science instrument optical design and monochromator commissioning, Rev. Sci. Instrum. 82, 093104 (2011).

[28] J. Amann, W. Berg, V. Blank, F.-J. Decker, Y. Ding, P. Emma, Y. Feng, J. Frisch, D. Fritz, J. Hastings et al., Demonstration of self-seeding in a hard-x-ray free-electron laser, Nat. Photonics 6, 693 (2012).

[29] A. A. Lutman, F.-J. Decker, J. Arthur, M. Chollet, Y. Feng, J. Hastings, Z. Huang, H. Lemke, H.-D. Nuhn, A. Marinelli, J. L. Turner, S. Wakatsuki, J. Welch, and D. Zhu, Demonstration of Single-Crystal Self-Seeded Two-Color X-ray Free-Electron Lasers, Phys. Rev. Lett. 113, 254801 (2014).

[30] D. Ratner, R. Abela, J. Amann, C. Behrens, D. Bohler, G. Bouchard, C. Bostedt, M. Boyes, K. Chow, D. Cocco et al., Experimental Demonstration of a Soft X-ray Self-Seeded Free-Electron Laser, Phys. Rev. Lett. 114, 054801 (2015).

[31] A. Marinelli, C. Pellegrini, L. Giannessi, and S. Reiche, Comparative study of nonideal beam effects in high gain harmonic generation and self-seeded free electron lasers, Phys. Rev. ST Accel. Beams 13, 070701 (2010).

[32] Z. Zhang, R. Lindberg, W. M. Fawley, Z. Huang, J. Krzywinski, A. Lutman, G. Marcus, and A. Marinelli, Microbunching-instability-induced sidebands in a seeded free-electron laser, Phys. Rev. Accel. Beams 19, 050701 (2016).

[33] C. Emma, A. Lutman, M. W. Guetg, J. Krzywinski, A. Marinelli, J. Wu, and C. Pellegrini, Ultra high brightness, femtosecond, coherent x-ray free-electron laser pulses (to be published). 